\title{
SUMO E3 Ligase PIASy Mediates High Glucose-Induced Activation of NF- $\kappa$ B Inflammatory Signaling in Rat Mesangial Cells
}

\author{
Wei Huang, ${ }^{1,2}$ Yaling Liang, ${ }^{1,3}$ Jianhua Dong, ${ }^{4}$ Luping Zhou, ${ }^{1,2}$ Chenlin Gao, ${ }^{1}$ \\ Chunxia Jiang, ${ }^{1}$ Meijuan Chen, ${ }^{3}$ Yang Long, ${ }^{1}$ and Yong $\mathrm{Xu}^{1,2}$ \\ ${ }^{1}$ Department of Endocrinology, The Affiliated Hospital of Southwest Medical University, Luzhou, Sichuan 646000, China \\ ${ }^{2}$ Collaborative Innovation Center for Prevention and Treatment of Cardiovascular Disease of Sichuan Province, \\ Southwest Medical University, Luzhou, China \\ ${ }^{3}$ Department of Geratology, The People's Hospital of Leshan, Leshan, Sichuan 614000, China \\ ${ }^{4}$ Department of Nephrology, The People's Hospital of Leshan, Leshan, Sichuan 614000, China
}

Correspondence should be addressed to Yang Long; xiaolong0325ly@163.com and Yong Xu; xywyll@aliyun.com

Received 20 March 2017; Revised 18 June 2017; Accepted 17 July 2017; Published 5 September 2017

Academic Editor: Dezheng Zhao

Copyright (c) 2017 Wei Huang et al. This is an open access article distributed under the Creative Commons Attribution License, which permits unrestricted use, distribution, and reproduction in any medium, provided the original work is properly cited.

\begin{abstract}
Background. Sumoylation is extensively involved in the regulation of NF- $\kappa$ B signaling. PIASy, as a SUMO E3 ligase, has been proved to mediate sumoylation of $\mathrm{I} \kappa \mathrm{B}$ kinase $\gamma(\mathrm{IKK} \gamma)$ and contribute to the activation of NF- $\kappa \mathrm{B}$ under genotoxic agent stimulation. However, the association of PIASy and NF- $\kappa \mathrm{B}$ signaling in the pathogenesis of diabetic nephropathy (DN) has not been defined. Methods. Rat glomerular mesangial cells (GMCs) were stimulated by high glucose; siRNA was constructed to silence the expression of PIASy; the expression of PIASy, SUMO isoforms (SUMO1, SUMO2/3), and NF- $\kappa \mathrm{B}$ signaling components was analyzed by Western blot; the interaction between IKK $\gamma$ and SUMO proteins was detected by coimmunoprecipitation; and the release of inflammatory cytokines MCP-1 and IL-6 was assayed by ELISA. Results. High glucose significantly upregulated the expression of PIASy, SUMO1, and SUMO2/3 in a dose- and timedependent manner $(P<0.05)$, induced the phosphorylation and sumoylation of $\operatorname{IKK} \gamma(P<0.05)$, and then triggered NF- $\kappa \mathrm{B}$ signaling whereas MCP-1 and IL-6 were released from GMCs $(P<0.05)$. Moreover, these high glucose-induced effects were observably reversed by siRNA-mediated knockdown of PIASy $(P<0.05)$. Conclusion. The SUMO E3 ligase PIASy mediates high glucose-induced activation of NF- $\kappa \mathrm{B}$ inflammatory signaling, suggesting that PIASy may be a potential therapeutic target of DN.
\end{abstract}

\section{Introduction}

Extensive research shows that systemic and local low-grade inflammation and release of proinflammatory cytokines resulting from activation of nuclear factor- $\kappa \mathrm{B}(\mathrm{NF}-\kappa \mathrm{B})$ signaling are involved in the development and progression of diabetes and diabetic nephropathy (DN) [1]. In fact, NF- $\kappa \mathrm{B}$ signaling plays critical roles in regulating immunity, cell survival, and expression of inflammatory cytokines. In the "resting" state, NF- $\kappa \mathrm{B}$ dimers are held inactive in the cytoplasm through association with $\mathrm{I} \kappa \mathrm{B}$ proteins. Inducing stimuli triggers the activation of the $\mathrm{I} \kappa \mathrm{B}$ kinase (IKK) complex, leading to phosphorylation, ubiquitination, and degradation of I $\kappa$ B proteins. Released NF- $\kappa$ B dimers translocate to the nucleus, bind to specific DNA sequences, and promote transcription of target genes. Thus, the core elements of the NF- $\kappa$ B pathway are the IKK complex, I $\kappa$ B proteins, and NF- $\kappa \mathrm{B}$ dimers [2].

Numerous regulatory posttranslational modifications (PTM), such as phosphorylation, acetylation, ubiquitination, and sumoylation, have been reported and shown to have effects on activating or inhibiting NF- $\kappa$ B induced by diverse 
agents. Sumoylation is a process of PTM by a relatively small polypeptides called small ubiquitin-like modifier (SUMO) [3]. Sumoylation has been shown to regulate each of its targets in a specific way by altering its conformation, stability, or interaction and localization properties. Our previous study demonstrated that high glucose obviously induced the expression of SUMO isoforms (SUMO1, SUMO2/3) in GMCs, subsequently stimulated degradation of $\mathrm{I} \kappa \mathrm{B} \alpha$, and triggered NF- $\kappa \mathrm{B}$ signaling by weakening the interaction between SUMO protein and $\mathrm{I} \kappa \mathrm{B} \alpha$ while promoting ubiquitination of $\mathrm{I} \kappa \mathrm{B} \alpha$ [4]. However, the association of SUMO ligases and NF- $\kappa \mathrm{B}$ signaling in $\mathrm{DN}$ has not been defined.

Sumoylation is a three-step process similar to ubiquitination that involves an E1-activating enzyme (Aos1/Uba2 heterodimer), an E2-conjugating enzyme (Ubc9), and E3 ligases, including the Ran-binding protein-2, Polycomb-2, and protein inhibitor of activated STAT (PIAS) proteins [5]. It is recently known that, in most cases, the final step of sumoylation reaction needs E3 ligases, which help to ensure substrate specificity and cell cycle dependence [6]. PIAS proteins were originally identified as repressors of the cytokineinduced STAT transcription factors. While PIAS proteins may act as E3 SUMO ligases, modulating the function of the target protein by adding SUMO tags, a growing body of evidence indicates that PIAS proteins may activate or repress transcription and play an important role in the regulation of transcription factors, including NF- $\kappa \mathrm{B}$, Smads, and p53 [7]. The mammalian PIAS protein family contains five members: PIAS1, PIAS3, PIASx $\alpha$, PIASx $\beta$, and PIASy; among PIAS proteins, PIASy can repress NF- $\kappa$ B activity in mouse keratinocytes through interaction with the RelA/p65 subunit of NF- $\kappa \mathrm{B}$, resulting in repressing the expression of CCL20 chemokine in response to TNF- $\alpha$ [8]. But the latest research proved that PIASy mediates IKK $\gamma$ sumoylation and NF- $\kappa \mathrm{B}$ activation in response to oxidative stress conditions [9], while the roles of PIASy in regulating NF- $\kappa \mathrm{B}$ inflammatory signaling induced by high glucose is still unclear. In this study, we detected the changes of PIASy, SUMO1, and SUMO2/3; NF- $\kappa \mathrm{B}$-related signaling molecules $(\mathrm{I} \kappa \mathrm{B} \alpha, \mathrm{p}-\mathrm{I} \kappa \mathrm{B} \alpha$, $\mathrm{p}-\mathrm{IKK} \gamma, \mathrm{IKK} \gamma, \mathrm{NF}-\kappa \mathrm{Bp} 65$, and p-NF- $\kappa \mathrm{Bp} 65)$; and downstream proinflammatory cytokines (MCP-1, IL-6) under high-glucose stress when the rGMCs were transfected with PIASy-siRNA or not, aiming to explore the roles of the SUMO E3 ligase PIASy on NF- $\kappa \mathrm{B}$ inflammatory signaling in the pathogenesis of $\mathrm{DN}$.

\section{Materials and Methods}

2.1. Cell Culture and Treatment. Rat GMCs (HBZY-1) were purchased from the Fudan IBS Cell Center and were cultured in Dulbecco's modified Eagle medium (DMEM, Hyclone, USA) containing $5.6 \mathrm{mmol} / \mathrm{L}$ glucose and $10 \%$ fetal bovine serum (FBS, Bovogen, Australia) at $37^{\circ} \mathrm{C}$ and $5 \% \mathrm{CO}_{2}$. GMCs were used for all experiments and were randomly divided into five groups: normal control group (NC group, with medium that contained $5.6 \mathrm{mmol} / \mathrm{L}$ glucose), $10 \mathrm{mmol} / \mathrm{L}$ glucose group (HG1 group, with medium that contained $10 \mathrm{mmol} / \mathrm{L}$ glucose), $20 \mathrm{mmol} / \mathrm{L}$ glucose group (HG2 group, with medium that contained $20 \mathrm{mmol} / \mathrm{L}$ glucose),
$30 \mathrm{mmol} / \mathrm{L}$ glucose group (HG3 group, with medium that contained $30 \mathrm{mmol} / \mathrm{L}$ glucose), and osmotic pressure group (OP group, with medium that contained $5.6 \mathrm{mmol} / \mathrm{L}$ glucose $+24.6 \mathrm{mmol} / \mathrm{L}$ mannitol as a control). After cells in each group were induced for $6,12,24,48$, and $72 \mathrm{~h}$, the culture supernatant was collected and the protein and mRNA were extracted for further study.

2.2. Small Interfering RNA Transfection. The PIASy duplex small interfering RNA (siRNA; RiboBio, China) was a pool of three-sequence siRNA targeting PIASy (number 1-sense: $5^{\prime}$-GCUGUAUGAGACUCGCUAUdTdT- $3{ }^{\prime}$ and antisense: $5^{\prime}$-AUAGCGAGCUCAUACAGCdTdT-3'; number 2-sense: $5^{\prime}$-GCAACUAUGGCAAGAGCUAdTdT- $3^{\prime}$ and antisense: $5^{\prime}$-UAGCUCUUGCCAUAGUUGCdTdT- ${ }^{\prime}$; ; and number 3-sense: $5^{\prime}$-GCAGCUUAUGACCAGCUCAdTdT- ${ }^{\prime}$ and antisense: $5^{\prime}$-UGAGCUGGUCAUAAGCUGCdTdT- $3^{\prime}$ ) or control siRNA (sense: $5^{\prime}$-UUCUCCGAACGUGUCACGU$3^{\prime}$ and anti-sense: $5^{\prime}$-ACGUGACACGUUCGGAGAA-3'). After the GMCs were transfected with PIASy-siRNA or control siRNA in serum-free Opti-MEM medium (Invitrogen, USA) with confluence of transfection (Roche) and siRNA at $37^{\circ} \mathrm{C}$ for $48 \mathrm{~h}$, siRNA-mediated knockdown of PIASy cells was then stimulated by $5.6 \mathrm{mmol} / \mathrm{L}$ glucose or $30 \mathrm{mmol} / \mathrm{L}$ glucose. At the end of each experiment, the cells and culture supernatant were then collected for ELISA and Western blotting analysis.

2.3. Protein Extraction and Western Blotting. Total protein was extracted from GMCs using a protein extraction kit (Kaiji, China). Proteins were separated by sodium dodecyl sulfate-polyacrylamide gel electrophoresis (SDS-PAGE) and transferred to a polyvinylidene difluoride (PVDF) membrane (Millipore). Immunoblotting was performed using antiPIASy antibody (mouse monoclonal antibody; 1:1000; Abcam, number ab211625), anti-SUMO1 antibody (rabbit monoclonal antibody; 1:800; Abcam, number 32058), antiSUMO2/3 antibody (rabbit polyclonal antibody; 1:600; Millipore, number $\mathrm{AB} 3876$ ), anti-I $\kappa \mathrm{B} \alpha$ antibody (mouse monoclonal antibody; 1:1000; CST, number 4814), anti$\mathrm{p}-\mathrm{I} \kappa \mathrm{B} \alpha$ antibody (ser32/36) (mouse monoclonal antibody; $1: 1000$; CST, number 9246), anti-NF- $\kappa$ Bp65 antibody (rabbit polyclonal antibody; 1:1000; Beyotime, number AF0246), anti-p-NF- $\kappa$ Bp65 antibody (ser536) (mouse monoclonal antibody; 1:2000; CST, number 13346), anti-p-IKK $\gamma$ antibody (ser85) (rabbit polyclonal antibody; 1:1000; Bioworld, number BS4597), anti-IKK $\gamma$ antibody (rabbit polyclonal antibody; 1:800; Santa Cruz, number sc-8830), and anti- $\beta$-actin antibody (mouse monoclonal antibody; $1: 2000$; Beyotime, number AF0003).

2.4. Enzyme-Linked Immunosorbent Assay (ELISA). Rat MCP-1 and IL-6 secretion was measured using MCP-1 and IL-6 ELISA kits (Westang Bio-Tech, China) according to the manufacturer's protocols. MCP-1 and IL- 6 protein levels were determined by comparing the samples to the standard curve generated by the kit.

2.5. Immunofluorescence. GMCs were grown on coverslips in 6-well plates. After overnight adherence, cells were incubated 
with $30 \mathrm{mmol} / \mathrm{L}$ high glucose or mannitol for $24 \mathrm{~h}$ as described above and then were fixed in $4 \%$ paraformaldehyde (Pierce Biotechnology, USA) and permeabilized in $0.25 \%$ Triton X-100 (Sigma, USA). Cells were blocked in 5\% goat serum, followed by incubation with anti-PIASy and antiSUMO1 or anti-SUMO2/3 antibody (dilution 1:100) overnight at $4^{\circ} \mathrm{C}$. After washing, cells were incubated with rhodamine- and fluorescein isothiocyanate-conjugated secondary antibodies (Bio-Synthesis) for $45 \mathrm{~min}$ in the dark. $4^{\prime}, 6^{\prime}$-Diamino-2-phenylindole (DAPI) was used to stain the nucleus in the cells. The coverslips were washed and imaged with a DMIRE2 laser scanning confocal microscope (Leica, Germany). The values of semiquantitative analysis for average intensity were assessed by Image-Pro Plus 6.0 software.

2.6. Immunoprecipitation and Immunoblot Analysis. Approximately $24 \mathrm{~h}$ after being treated with media that contained high glucose and mannitol, the cells were harvested. Ice-cold immunoprecipitation lysis/wash buffer was added using a coimmunoprecipitation kit (Pierce Biotechnology, USA) with protease inhibitors (Roche, USA). The cell lysates were clarified by centrifugation at $13,000 \mathrm{~g}$ for $10 \mathrm{~min}$ at $4^{\circ} \mathrm{C}$, and the supernatants were subjected to immunoprecipitation. The supernatants were incubated with monoclonal anti-IKK $\gamma$ antibody (rabbit polyclonal antibody; Santa Cruz, number sc-8830) and normal rabbit immunoglobulin G (IgG, Beyotime, China) for $12 \mathrm{~h}$ at $4^{\circ} \mathrm{C}$. After incubation, protein A/G Sepharose was used for precipitation. The beads were washed with $1 \times$ conditioning buffer. The antigenantibody complexes were collected, washed, and boiled in $2 \times$ lane marker nonreducing sample buffer. For the immunoblot analysis, proteins were probed with anti-SUMO1 antibody (rabbit monoclonal antibody; 1:800; Abcam, number 211625) or anti-SUMO2/3 antibody (rabbit polyclonal antibody; 1:600; Millipore, number AB3876).

2.7. Data Analysis. All data obtained from at least three independent experiments were expressed as the means \pm standard deviation (SD), and between-group comparisons were analyzed using one-way analysis of variance (ANOVA), followed by the LSD post hoc test for multiple comparisons (SPSS 17 software). $P<0.05$ was considered significant.

\section{Results}

3.1. High Concentrations of Glucose Induce PIASy and SUMO Isoform Expression and Colocalization in GMCs. Compared to the NC group, the expression of PIASy was significantly induced following $6,12,24,48$, and $72 \mathrm{~h}$ of exposure to $30 \mathrm{mmol} / \mathrm{L}$ glucose $(P<0.05)$; the highest relative expression of PIASy protein was observed after stimulation of $72 \mathrm{~h}$. Moreover, PIASy protein levels were also significantly enhanced by different concentrations of glucose at $24 \mathrm{~h}$ $(P<0.05)$; the highest relative expression of PIASy was detected in the $30 \mathrm{mmol} / \mathrm{L}$ glucose group. A significant difference was also found between the OP group and NC group. However, PIASy protein levels were significantly decreased in the OP group compared with the $20 \mathrm{mmol} / \mathrm{L}$ and $30 \mathrm{mmol} / \mathrm{L}$ glucose groups $(P<0.05)$ (Figure $1(\mathrm{a}))$, suggesting that high glucose concentration increased the expression of PIASy in a time- and dose-dependent manner, and osmotic pressure had a little effect on the high glucoseinduced PIASy expression. Consistent with PIASy, the expression of SUMO isoforms (SUMO1 and SUMO2/3) was significantly increased by high glucose in a time- and dose-dependent manner $(P<0.05)$ (Figure $1(\mathrm{~b}))$. Moreover, the merged images of immunofluorescence showed that PIASy colocalized with SUMO1 (Figure 1(c)) or SUMO2/3 (Figure 1(d)) in the nucleus, compared to the NC group and OP group; the average intensity of these proteins was strongly enhanced in the $30 \mathrm{mmol} / \mathrm{L}$ glucose group $(P<0.05)$. These results suggested that high glucose increased the expression of PIASy, SUMO1, and SUMO2/3, while inducing the colocalization of PIASy and SUMO1 or SUMO2/3 in the nucleus of GMCs.

3.2. High Glucose Induced the Phosphorylation and Sumoylation of IKK $\gamma$ in GMCs. Compared to the NC group, there was no significant change in the expression of IKK $\gamma$ following different concentrations of high-glucose treatment, while high glucose, especially $30 \mathrm{mmol} / \mathrm{L}$ glucose, observably induced the expression of phosphorylation of IKK $\gamma$ after $24 \mathrm{~h}$ stimulation $(P<0.05)$ (Figure $2(\mathrm{a}))$. Next, we performed the immunoprecipitation and immunoblot analysis to determine whether SUMO is involved in IKK $\gamma$ sumoylation in GMCs. As shown in Figure 2(b), IKK $\gamma$ antibodies immunoprecipitated a polypeptide of about $72 \mathrm{kD}$ that was recognized by the SUMO-specific antibody. The results showed that SUMO1 and SUMO2/3 were coimmunoprecipitated with IKK $\gamma$, and the SUMO-induced modification of IKK $\gamma$ was detected on endogenously expressed proteins, suggesting that SUMO and IKK $\gamma$ were able to form a complex in GMCs. To determine whether IKK $\gamma$ sumoylation is affected by high glucose, we assessed the effect of $30 \mathrm{mmol} / \mathrm{L}$ glucose and mannitol treatment on IKK $\gamma$ sumoylation. Interestingly, the interaction between IKK $\gamma$ and SUMO1 or SUMO2/3 was increased by high glucose $(P<0.05)$ (Figure $2(\mathrm{c}))$, but osmotic pressure had a little effect on the high glucoseinduced IKK $\gamma$ sumoylation. Taken together, these data indicated that phosphorylation and sumoylation of IKK $\gamma$ were induced by high glucose in GMCs.

3.3. High Glucose Significantly Activated NF- $\kappa B$ Inflammatory Signaling by Degradation of $I \kappa B \alpha$. The relative expression of $\mathrm{p}-\mathrm{I} \kappa \mathrm{B} \alpha, \mathrm{p}-\mathrm{NF}-\kappa \mathrm{Bp} 65$, and NF- $\kappa \mathrm{Bp} 65$ was markedly increased following $6,12,24,48$, and $72 \mathrm{~h}$ of exposure to $30 \mathrm{mmol} / \mathrm{L}$ glucose $(P<0.05)$ (Figure $3(\mathrm{a}))$. Furthermore, $\mathrm{p}-\mathrm{I} \kappa \mathrm{B} \alpha, \mathrm{p}-\mathrm{NF}-\kappa \mathrm{Bp} 65$, and NF- $\kappa \mathrm{Bp} 65$ were also significantly induced by different concentrations of high glucose, especially in the $30 \mathrm{mmol} / \mathrm{L}$ glucose group $(P<0.05)$; the addition of $30 \mathrm{mmol} / \mathrm{L}$ mannitol to normal glucose did not change the expression of these proteins $(P>0.05)$, indicating that the high glucose-induced activation of NF- $\kappa \mathrm{B}$ signaling molecules was not an osmotic effect (Figure 3(b)). However, the expression of $\mathrm{I} \kappa \mathrm{B} \alpha$ was significantly attenuated by high glucose in a time- and dose-dependent manner $(P<0.05)$ (Figures 3(a) and 3(b)). Consistent with the activation of signaling molecules, the release of inflammatory 

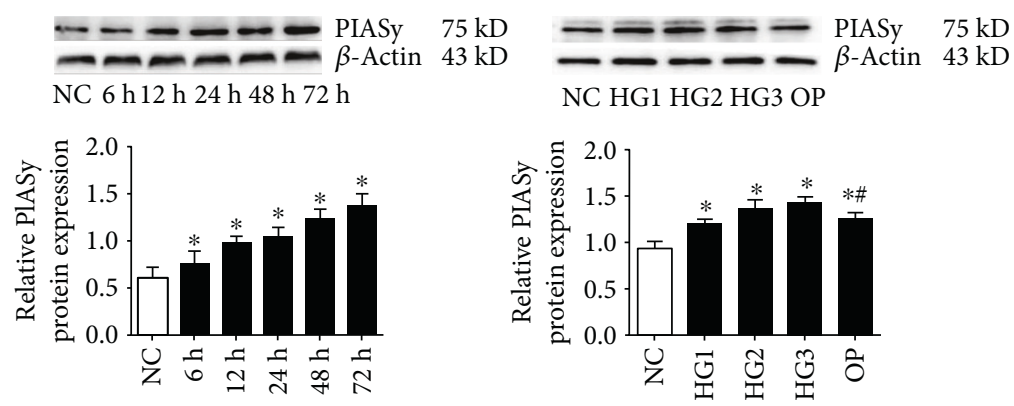

(a)
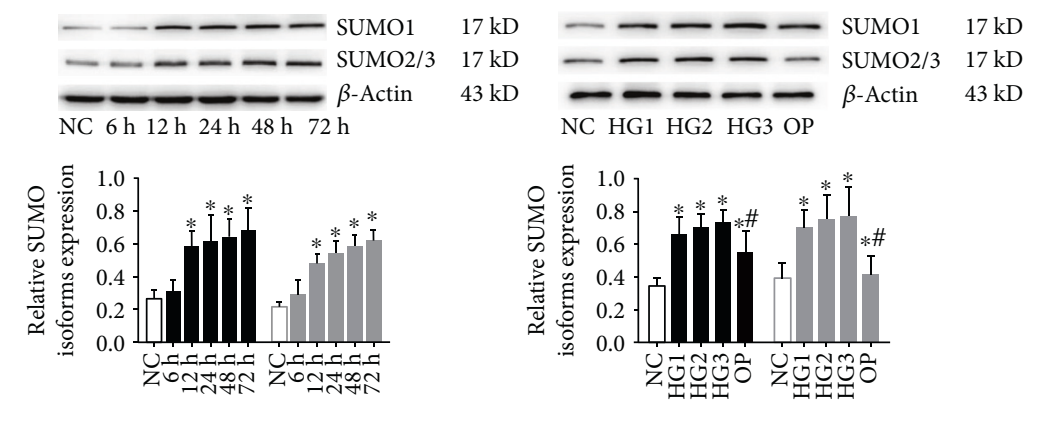

- SUMO1 protein
- SUMO2/3 protein

$$
\begin{aligned}
& \text { - SUMO1 protein } \\
& \text { - SUMO2/3 protein }
\end{aligned}
$$

(b)
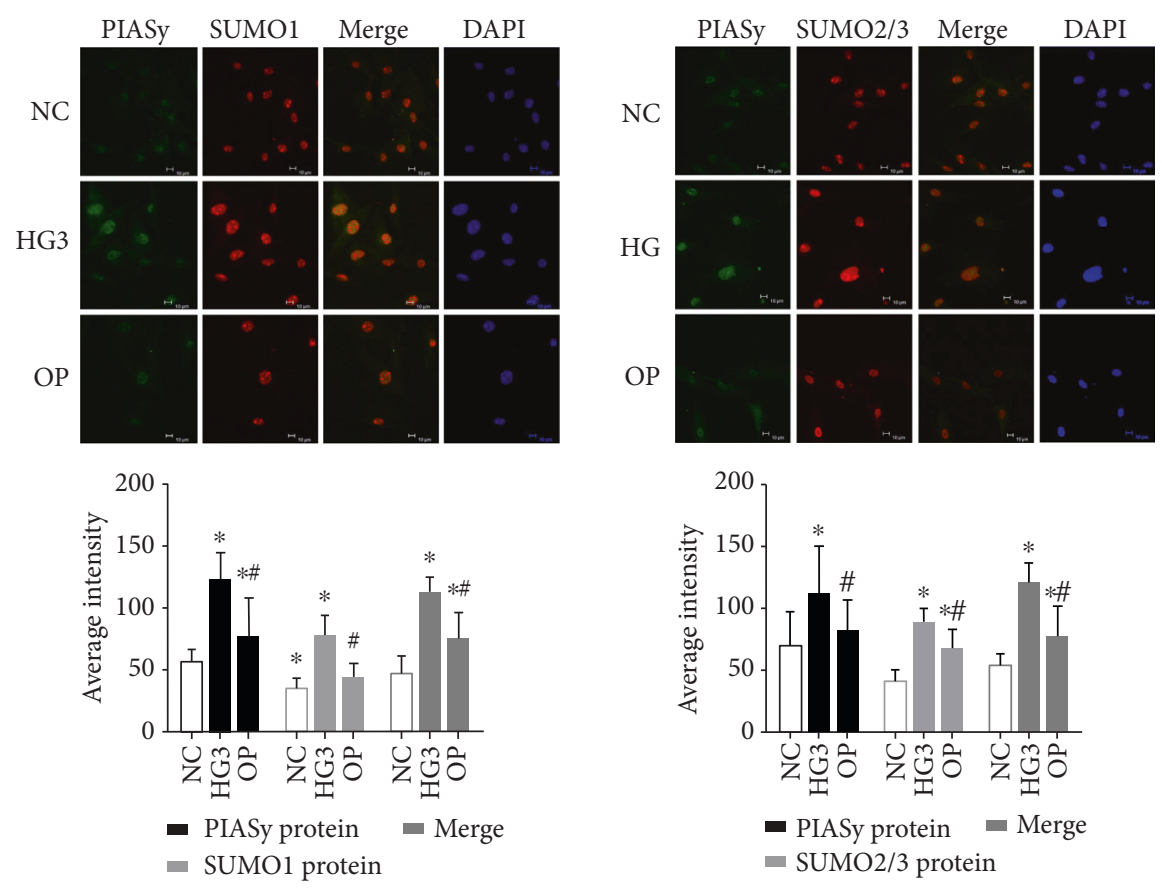

(c)

(d)

FIgURE 1: The expression of PIASy and SUMO isoforms (SUMO1, SUMO2/3) was induced by various times and various glucose concentrations. GMCs were treated with $30 \mathrm{mmol} / \mathrm{L}$ high glucose for $6,12,24,48$, and $72 \mathrm{~h}$ or the indicated concentrations of glucose or mannitol for $24 \mathrm{~h}$; the protein expression of PIASy (a) and SUMO isoforms (SUMO1, SUMO2/3) (b) in lysates of cells was detected by Western blotting. Data were normalized with respect to $\beta$-actin and are expressed as mean $\pm \operatorname{SD}(n=5)$. The gray graphs confirmed these trends. Immunofluorescence was performed to determine intensity and subcellular localization of PIASy and SUMO1 (c) or SUMO2/3 (d) after GMC treatment with $30 \mathrm{mmol} / \mathrm{L}$ high glucose or mannitol for $24 \mathrm{~h}(400 \mathrm{x})$. The merged images of PIASy and SUMO1 or SUMO2/3 stainings in each group were shown, and DAPI was used to stain the nucleus in the cells. The values of semiquantitative analysis for average intensity were assessed, and the gray graphs confirmed these trends. ${ }^{*} P<0.05$ compared with the NC group, ${ }^{\#} P<0.05$ compared with the $30 \mathrm{mmol} / \mathrm{L}$ high glucose stimulation group. 

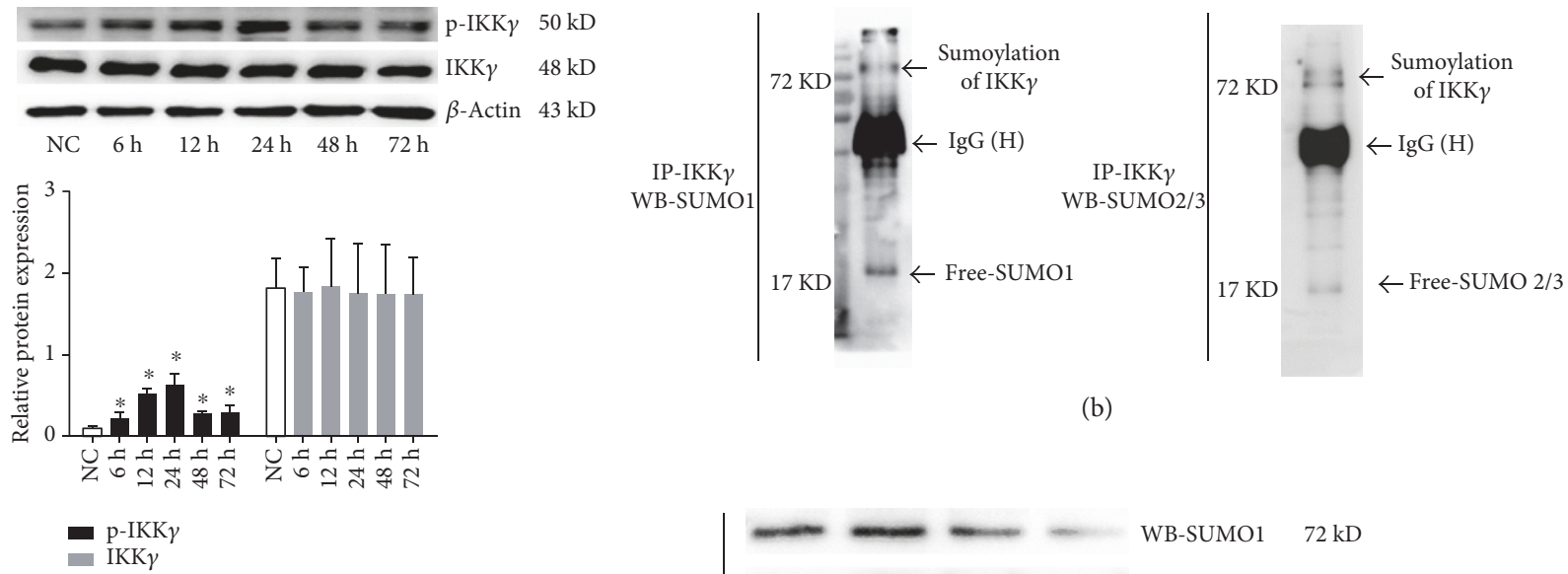

(b)
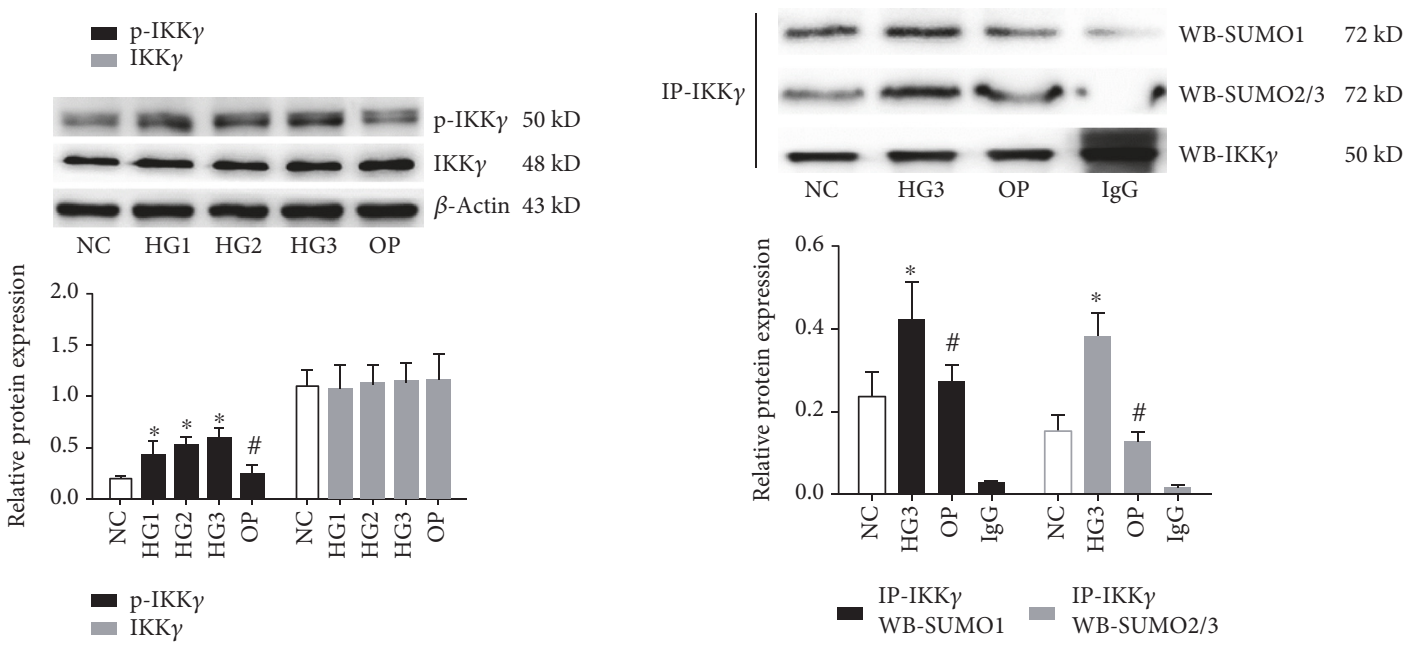

(a)

(c)

FIgURE 2: The phosphorylation and sumoylation of IKK $\gamma$ were induced by high glucose. (a) The protein expression of $\mathrm{p}$-IKK $\gamma$ and IKK $\gamma$ in lysates of GMCs was detected after high glucose challenge for various times and various glucose concentrations by Western blotting. (b) IKK $\gamma$ sumoylation was detected by immunoprecipitation (IP) with anti-IKK $\gamma$ antibody followed by Western blotting with anti-SUMO1 or antiSUMO2/3 antibody. IKK $\gamma$ was conjugated with SUMO in GMCs. IgG (H) marks the IgG heavy chain. (c) GMCs were treated with $30 \mathrm{mmol} / \mathrm{L}$ high glucose or mannitol for $24 \mathrm{~h}$. Anti-IKK $\gamma$ immunoprecipitates were subjected to immunoblotting with anti-SUMO1 or anti-SUMO2/3 antibody to detect IKK $\gamma$ and SUMO-IKK $\gamma$ proteins; normal IgG antibody was used as a negative control. IKK $\gamma$ that was sumoylated by SUMO1 or SUMO2/3 was induced by high glucose. The gray graphs confirmed these trends. Data are expressed as mean $\pm \mathrm{SD}(n=3) .{ }^{*} P<0.05$ compared with the NC group, ${ }^{\#} P<0.05$ compared with the $30 \mathrm{mmol} / \mathrm{L}$ high glucose stimulation group.

cytokines MCP-1 and IL-6 from GMCs was also obviously increased by high glucose in a time- and dose-dependent manner $(P<0.05)$ (Figure $3(c))$. These data suggested that high glucose, but not osmotic pressure, induced the degradation of $\mathrm{I} \kappa \mathrm{B} \alpha$ and activated NF- $\kappa \mathrm{B}$ inflammatory signaling.

3.4. High Glucose-Induced Sumoylation of IKK $\gamma$ and NF- $\kappa B$ Activation Were Significantly Reversed by siRNA-Mediated Knockdown of PIASy. PIASy expression was successfully inhibited by PIASy-siRNA $(P<0.05)$, especially by PIASysiRNA-3, which was chosen for subsequent experiments (Figure 4(a)). The interaction between IKK $\gamma$ and SUMO1 or SUMO2/3 induced by high glucose was reversed by siRNA-PIASy-3 (Figure 4(b)). Indeed, siRNA-mediated knockdown of PIASy also markedly attenuated the phosphorylation of IKK $\gamma$ and $\mathrm{I} \kappa \mathrm{B} \alpha$, inhibited the degradation of $\mathrm{I} \kappa \mathrm{B} \alpha$, and reversed the activation of $\mathrm{NF}-\kappa \mathrm{B}$ induced by $30 \mathrm{mmol} / \mathrm{L}$ glucose for $24 \mathrm{~h}(P<0.05)$ (Figure $4(\mathrm{c}))$. To investigate whether the downstream proinflammatory cytokines of NF- $\kappa \mathrm{B}$ signaling may be inhibited by siRNA-mediated knockdown of PIASy, as expected, elevated MCP-1 and IL6 release from GMCs was blunted by PIASy gene silencing $(P<0.05) \quad$ (Figure $4(\mathrm{~d}))$. These results suggested that siRNA-mediated knockdown of PIASy inhibited the sumoylation of IKK $\gamma$ and the activation of NF- $\kappa$ B inflammatory signaling; in other words, the SUMO E3 ligase PIASy plays an important role in the activation of $\mathrm{NF}-\kappa \mathrm{B}$ signaling induced by high glucose.

\section{Discussion}

GMC proliferation and hypertrophy, ECM accumulation, and consequent renal fibrosis induced by high glucose, AGEs, or LPS have been recognized as major pathogenic events in the progression of renal failure in DN [10]. Numerous evidences demonstrate that the activation of NF- $\kappa \mathrm{B}$ in GMCs plays a central regulatory role in the expression of various inflammatory cytokines (such as MCP-1 and IL-6) involved in the occurrences of DN [11]. In agreement with previous reports, our present results indicate that high glucose may 

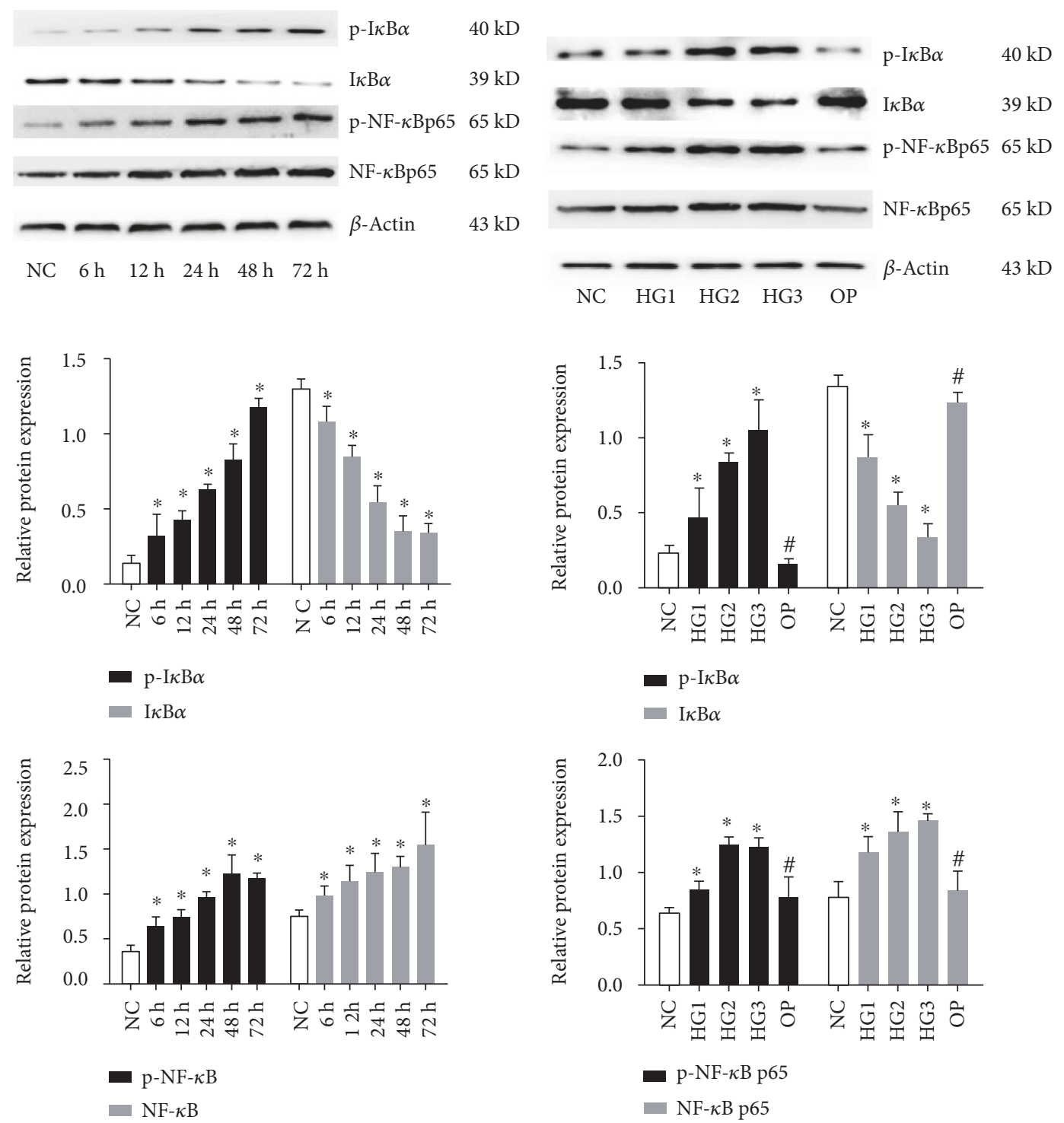

(a)

(b)
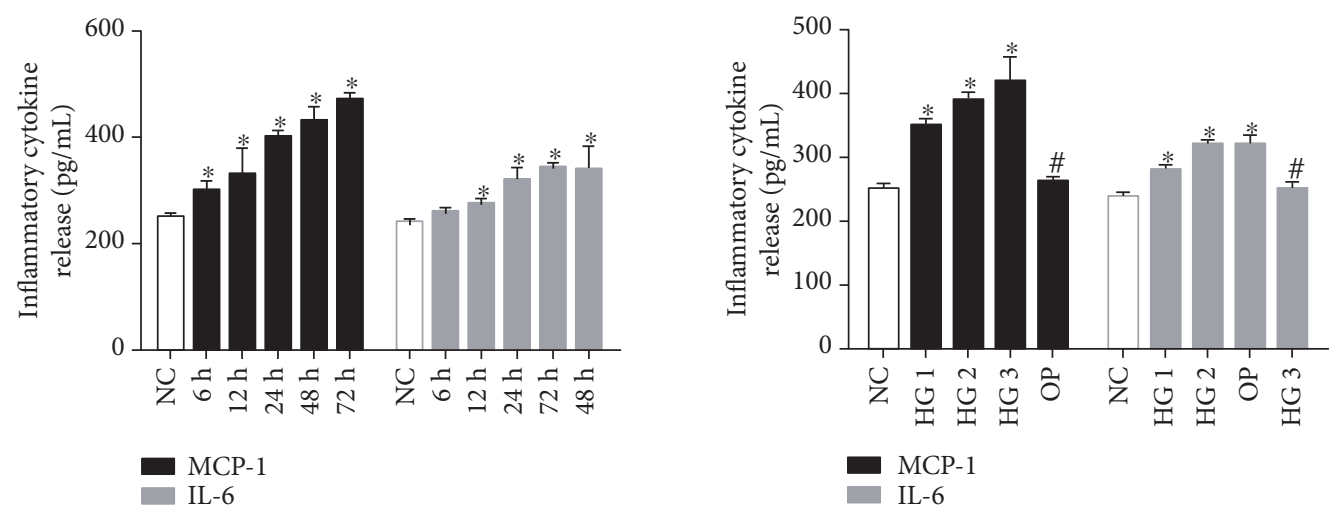

(c)

FIGURE 3: High glucose significantly activated the NF- $\kappa \mathrm{B}$ inflammatory signaling by degradation of $\mathrm{I} \kappa \mathrm{B} \alpha$. GMCs were treated with $30 \mathrm{mmol} / \mathrm{L}$ high glucose for $6,12,24,48$, and $72 \mathrm{~h}$ (a) or the indicated concentrations of glucose or mannitol (b) for $24 \mathrm{~h}$; the expression of p-I $\kappa \mathrm{B} \alpha$, $\mathrm{I} \kappa \mathrm{B} \alpha, \mathrm{p}-\mathrm{NF}-\kappa \mathrm{B}$, and NF- $\kappa \mathrm{B}$ in lysates of cells was detected by Western blotting. Data were normalized with respect to $\beta$-actin and are expressed as mean \pm SD $(n=3)$. The gray graphs confirmed these trends. MCP-1 and IL- 6 protein levels in the cell culture supernatant were determined by ELISA-based quantification (c). ${ }^{*} P<0.05$ compared with the NC group, ${ }^{\#} P<0.05$ compared with the 30 mmol/L high glucose stimulation group. 

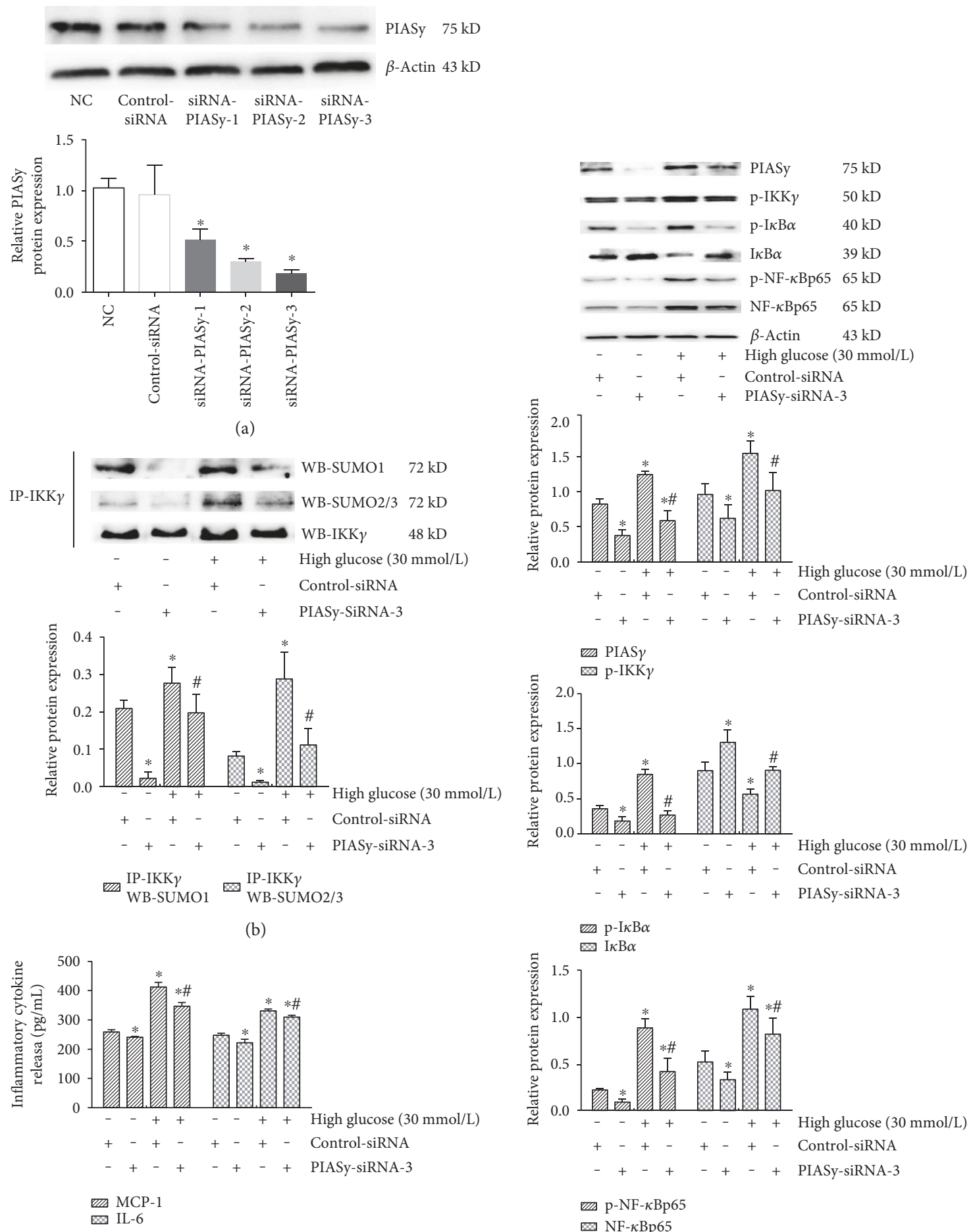

(d)

(c)

FIGURE 4: High glucose-mediated activation of NF- $\kappa$ B inflammatory signaling was reversed by siRNA-PIASy. (a) Western blotting analysis of protein expression after siRNA-mediated knockdown of PIASy has proven effective in silencing target genes of PIASy by siRNA-PIASy-3. (b) Immunoprecipitation and immunoblot analysis of the interaction between IKK $\gamma$ and SUMO1 or SUMO2/3 induced by high glucose was reversed by siRNA-PIASy-3. (c) Western blotting analysis showed that the changes in PIASy, p-IKK $\gamma, \mathrm{p}-\mathrm{I} \kappa \mathrm{B} \alpha, \mathrm{I} \kappa \mathrm{B} \alpha, \mathrm{p}-\mathrm{NF}-\kappa \mathrm{Bp} 65$, and NF- $\kappa$ Bp65 expression after high glucose challenge were significantly reversed by siRNA-PIASy-3. (d) ELISA-based quantification indicated that high glucose-induced release of MCP-1 and IL-6 from GMCs was blunted by siRNA-PIASy-3. The results are presented as mean $\pm \mathrm{SD}(n=5)$; the gray graphs confirmed these trends. ${ }^{*} P<0.05$ compared with the NC group, ${ }^{\#} P<0.05$ compared with the $30 \mathrm{mmol} / \mathrm{L}$ high glucose stimulation group. 
be involved in the pathogenesis of $\mathrm{DN}$ via manifesting upregulation of phosphorylation of $\mathrm{I} \kappa \mathrm{B} \alpha$, which results in $\mathrm{I} \kappa \mathrm{B} \alpha$ degradation and activation of NF- $\kappa \mathrm{B}$. Our experimental results also showed that the release of MCP-1 and IL- 6 from GMCs was significantly upregulated by high glucose in a dose-dependent and time-dependent manner, suggesting that high glucose induces a renal inflammatory state. Therefore, novel therapeutic strategies that target gene-special regulators of $\mathrm{NF}-\kappa \mathrm{B}$ may prove to be more efficient for the treatment of $\mathrm{DN}$.

Sumoylation plays an important role in multiple biological processes, such as protein interactions, protein stability, nuclear-cytoplasmic trafficking, transcriptional regulation, DNA repair, and cellular signaling pathways [12, 13]. Multiple signal transduction molecules of the NF- $\kappa \mathrm{B}$ pathway, such as $\mathrm{I} \kappa \mathrm{B} \alpha, \mathrm{IKK}, \mathrm{RelA}$, and $\mathrm{P} 100$, can be modified by SUMO $[8,9,14]$. SUMO1 can mediate the sumoylation of $\mathrm{I} \kappa \mathrm{B} \alpha$, resulting in sustaining the stability of $\mathrm{I} \kappa \mathrm{B} \alpha$, in case of degradation, and inhibiting inflammatory NF- $\kappa \mathrm{B}$ pathway activation [15]. Overexpression of SUMO4 also contributes to enhancing the sumoylation of $\mathrm{I} \kappa \mathrm{B} \alpha$ and regulating NF$\kappa \mathrm{B}$ activation under external stimulus stimulation, which is considered to be strongly associated with type 1 diabetes [16]. SUMO E3 ligases play a key role in sumoylation and ensure substrate specificity and cell cycle dependence in response to different stresses. Previous studies reported that PIASy is located predominantly in the nucleus and interacts with various transcription factors; however, it has also been reported that PIASy interacts with cytoplasmic proteins, such as axin [17]. Studies showed that PIASy cooperated with PIAS1 to regulate the specificity and magnitude of NF- $\kappa \mathrm{B}-$ mediated gene activation [18]. Consistent with previous results, our data showed that high glucose increased the protein expression of SUMO1 and SUMO2/3 in a time- and dose-dependent manner; furthermore, high glucose also upregulated the expression of PIASy and induced the colocalization of PIASy and SUMO1 or SUMO2/3 in the nucleus of GMCs; osmotic stress had a little effect on the expression of SUMO and PIASy proteins, suggesting that PIASy, SUMO1, and SUMO2/3, which may be considered cellular stress proteins, may play an important role in the regulation of the NF$\kappa \mathrm{B}$ pathway in response to high glucose during renal injury.

Under oxidative stress, PIASy mediates transglutaminase (TG2) sumoylation and inhibits TG2 ubiquitination and proteasome degradation, leading to sustenance of TG2 activation, which prevents $\mathrm{I} \kappa \mathrm{B} \alpha$ sumoylation and results in NF$\kappa \mathrm{B}$ activation and an uncontrolled inflammatory response, but SUMO1 or PIASy gene silencing can induce TG2 degradation and restore $\mathrm{I} \kappa \mathrm{B} \alpha$ sumoylation, thus switching off inflammation [19]. Our previous studies have already demonstrated that high glucose decreased sumoylation of $\mathrm{I} \kappa \mathrm{B} \alpha$ by weakening the interaction between SUMO2/3 and $\mathrm{I} \kappa \mathrm{B} \alpha$, suggesting that the stability of SUMO-I $\kappa \mathrm{B} \alpha$ plays a predominance function in regulating NF- $\kappa \mathrm{B}$ inflammatory signaling in response to high glucose [4]. Here, our present results suggested that high glucose-induced upregulation of PIASy could affect the stability of $\mathrm{I} \kappa \mathrm{B} \alpha$, leading to the degradation of $\mathrm{I} \kappa \mathrm{B} \alpha$ and activation of $\mathrm{NF}-\kappa \mathrm{B}$ inflammatory signaling, but the underlying mechanisms are yet unidentified.
IKK $\gamma$, known as NEMO (NF- $\kappa \mathrm{B}$ essential modulator), a noncatalytic subunit of the IKK complex, plays an essential regulatory role for the $\mathrm{NF}-\kappa \mathrm{B}$ canonical pathway. A series of publications now provide information about ubiquitination, sumoylation, or phosphorylation of IKK $\gamma$ which regulates its function in the IKK complex. These modifications might also regulate a cytosolic pool of free IKK $\gamma$ that controls the activation of NF- $\kappa \mathrm{B}$ induced by genotoxic stress $[20,21]$. In this study, we performed an experiment to determine whether high glucose is involved in IKK $\gamma$ sumoylation in GMCs. Our data showed that no significant change in the protein expression of IKK $\gamma$ was found in GMCs induced by high glucose and high osmotic pressure, while the expression of p-IKK $\gamma$ and the interaction between IKK $\gamma$ and SUMO1 or SUMO2/3 were observably induced by high glucose, suggesting that high glucose was involved in phosphorylation and sumoylation of IKK $\gamma$. However, as a SUMO ligase, the role of PIASy in this process needs to be further confirmed.

Previous research revealed that PIASy preferentially stimulates site-selective modification of IKK $\gamma$ by SUMO1, but not SUMO2 and SUMO3, in vitro. PIASy-IKK $\gamma$ interaction is increased by genotoxic stress and oxidative stress; siRNA-PIASy inhibits IKK $\gamma$ sumoylation and NF- $\kappa \mathrm{B}$ activation, and overexpression of PIASy enhances these events [9]. Further studies have found that activated poly(ADPribose)-polymerase-1 (PARP-1) and a PAR-binding motifs (PARBM) in PIASy are required to trigger IKK $\gamma$ sumoylation, which in turn permits IKK and NF- $\kappa$ B activation, as well as NF- $\kappa \mathrm{B}$-regulated resistance to apoptosis [22], and exported sumoylated IKK $\gamma$ acts as a substrate. IKK $\gamma$ monoubiquitination is a prerequisite for genotoxic IKK and NF- $\kappa \mathrm{B}$ activation but also promotes cytokine signaling [23]. However, whether PIASy participates in high glucose-induced $\mathrm{NF}-\kappa \mathrm{B}$ inflammatory signaling in GMCs has not been defined. Here, our results firstly demonstrate that high glucose-induced phosphorylation and sumoylation of IKK $\gamma$ were reversed by siRNA-PIASy. Moreover, degradation of $\mathrm{I} \kappa \mathrm{B} \alpha$, activation of $\mathrm{NF}-\kappa \mathrm{B}$, and release of downstream inflammatory cytokines MCP-1 and IL-6 from GMCs induced by high glucose were blunted by siRNA-PIASy. Based on our findings, we suggest a new model for the activation of NF- $\kappa$ B inflammatory signaling: exposure of the GMCs to high glucose results in the overexpression of the SUMO E3 ligase PIASy and SUMO proteins, which causes the SUMO proteins to bind to IKK $\gamma$, mediating the phosphorylation and sumoylation of IKK $\gamma$; the subsequent degradation of $\mathrm{I} \kappa \mathrm{B} \alpha$ and activation of NF- $\kappa \mathrm{B}$ in turn result in the processing of MCP-1 and IL- 6 release from GMCs, eventually promoting the renal low-grade inflammation. In other words, these combined results firstly reveal that upregulation of PIASy may play an important role in NF- $\kappa \mathrm{B}$ activation in the pathogenesis of DN. However, our studies by no means rule out other potential mechanisms by which sumoylation may regulate the NF- $\kappa \mathrm{B}$ pathway, in view of the fact that the regulatory mechanisms of NF- $\kappa \mathrm{B}$ are extremely complex and the types of SUMO E3 ligases are diverse.

In summary, our study has firstly demonstrated that high glucose increased the expression of PIASy in a doseand time-dependent manner, subsequently induced the 
phosphorylation and sumoylation of IKK $\gamma$ and then degradation of $\mathrm{I} \kappa \mathrm{B} \alpha$, and activated the NF- $\kappa \mathrm{B}$ inflammatory signaling in GMCs, which can be switched off by siRNAmediated knockdown of PIASy. The present results support the hypothesis that the SUMO E3 ligase PIASy mediates high glucose-induced activation of NF- $\kappa \mathrm{B}$ inflammatory signaling, suggesting that PIASy may be a potential therapeutic target of DN.

\section{Disclosure}

Yaling Liang is the co-first author.

\section{Conflicts of Interest}

The authors declare that they have no competing interests regarding the publication of this paper.

\section{Acknowledgments}

This research was supported by the collaborative Innovation Center for Prevention and Treatment of Cardiovascular Disease of Sichuan Province. The authors gratefully acknowledge the Clinical Center Laboratory for the technical assistance.

\section{References}

[1] Z. Zheng and F. Zheng, "Immune cells and inflammation in diabetic nephropathy," Journal of Diabetes Research, vol. 2016, Article ID 1841690, 10 pages, 2016.

[2] M. S. Hayden and S. Ghosh, "NF- $\kappa$ B, the first quarter-century: remarkable progress and outstanding questions," Genes \& Development, vol. 26, no. 3, pp. 203-234, 2012.

[3] C. Gao, W. Huang, K. Kanasaki, and Y. Xu, "The role of ubiquitination and sumoylation in diabetic nephropathy," BioMed Research International, vol. 2014, Article ID 160692, 11 pages, 2014.

[4] W. Huang, L. Xu, X. Zhou et al., "High glucose induces activation of NF- $\kappa$ B inflammatory signaling through $\mathrm{I} \kappa \mathrm{B} \alpha$ sumoylation in rat mesangial cells," Biochemical and Biophysical Research Communications, vol. 438, no. 3, pp. 568-574, 2013.

[5] J. Long, I. Matsuura, D. He, G. Wang, K. Shuai, and F. Liu, "Repression of Smad transcriptional activity by PIASy, an inhibitor of activated STAT," Proceedings of the National Academy of Sciences of the United States of America, vol. 100, no. 17, pp. 9791-9796, 2003.

[6] A. M. Andreou and N. Tavernarakis, "Roles for SUMO modification during senescence," Advances in Experimental Medicine and Biology, vol. 694, pp. 160-171, 2010.

[7] K. Shuai and B. Liu, "Regulation of gene-activation pathways by PIAS proteins in the immune system," Nature Reviews Immunology, vol. 5, no. 8, pp. 593-605, 2005.

[8] Y. Liu, J. P. Lagowski, S. Gao, J. H. Raymond, C. R. White, and M. F. Kulesz-Martin, "Regulation of the psoriatic chemokine CCL20 by E3 ligases Trim32 and Piasy in keratinocytes," The Journal of Investigative Dermatology, vol. 130, no. 5, pp. 1384-1390, 2010.

[9] A. M. Mabb, S. M. Wuerzberger-Davis, and S. Miyamoto, "PIASy mediates NEMO sumoylation and NF-kappaB activation in response to genotoxic stress," Nature Cell Biology, vol. 8, no. 9, pp. 986-993, 2006.

[10] S. Tang, C. Gao, Y. Long et al., "Maresin 1 mitigates high glucose-induced mouse glomerular mesangial cell injury by inhibiting inflammation and fibrosis," Mediators of Inflammation, vol. 2017, Article ID 2438247, 11 pages, 2017.

[11] X. M. Wu, Y. B. Gao, F. Q. Cui, and N. Zhang, "Exosomes from high glucose-treated glomerular endothelial cells activate mesangial cells to promote renal fibrosis," Biology Open, vol. 5, no. 4, pp. 484-491, 2015.

[12] K. Eifler and A. C. Vertegaal, "SUMOylation-mediated regulation of cell cycle progression and cancer," Trends in Biochemical Sciences, vol. 40, no. 12, pp. 779-793, 2015.

[13] K. E. Coleman and T. T. Huang, "How SUMOylation finetunes the Fanconi anemia DNA repair pathway," Frontiers in Genetics, vol. 7, p. 61, 2016.

[14] J. Vatsyayan, G. Qing, G. Xiao, and J. Hu, "SUMO1 modification of NF-kappaB2/p100 is essential for stimuli-induced p100 phosphorylation and processing," EMBO Reports, vol. 9, no. 9, pp. 885-890, 2008.

[15] J. M. Desterro, M. S. Rodriguez, and R. T. Hay, "SUMO-1 modification of IkappaBalpha inhibits NF-kappaB activation," Molecular Cell, vol. 2, no. 2, pp. 233-239, 1998.

[16] C. Y. Wang, P. Yang, M. Li, and F. Gong, "Characterization of a negative feedback network between SUMO4 expression and NFkappaB transcriptional activity," Biochemical and Biophysical Research Communications, vol. 381, no. 4, pp. 477-481, 2009.

[17] H. L. Rui, E. Fan, H. M. Zhou, Z. Xu, Y. Zhang, and S. C. Lin, "SUMO-1 modification of the C-terminal KVEKVD of axin is required for JNK activation but has no effect on Wnt signaling," The Journal of Biological Chemistry, vol. 277, no. 45, pp. 42981-42986, 2002.

[18] S. Tahk, B. Liu, V. Chernishof, K. A. Wong, H. Wu, and K. Shuai, "Control of specificity and magnitude of NF-kappa $\mathrm{B}$ and STAT1-mediated gene activation through PIASy and PIAS1 cooperation," Proceedings of the National Academy of Sciences of the United States of America, vol. 104, no. 28, pp. 11643-11648, 2007.

[19] A. Luciani, V. R. Villella, A. Vasaturo et al., "SUMOylation of tissue transglutaminase as link between oxidative stress and inflammation," Journal of Immunology, vol. 183, no. 4, pp. 2775-2784, 2009.

[20] T. T. Huang, S. M. Wuerzberger-Davis, Z. H. Wu, and S. Miyamoto, "Sequential modification of NEMO/IKKgamma by SUMO-1 and ubiquitin mediates NF-kappaB activation by genotoxic stress," Cell, vol. 115, no. 5, pp. 565-576, 2003.

[21] H. Sebban, S. Yamaoka, and G. Courtois, "Posttranslational modifications of NEMO and its partners in NF-kappaB signaling," Trends in Cell Biology, vol. 16, no. 11, pp. 569-577, 2006.

[22] M. Stilmann, M. Hinz, S. C. Arslan, A. Zimmer, V. Schreiber, and C. Scheidereit, "A nuclear poly(ADP-ribose)-dependent signalosome confers DNA damage-induced IkappaB kinase activation," Molecular Cell, vol. 36, no. 3, pp. 365-378, 2009.

[23] M. Hinz, M. Stilmann, S. Ç. Arslan, K. K. Khanna, G. Dittmar, and C. Scheidereit, "A cytoplasmic ATM-TRAF6-cIAP1 module links nuclear DNA damage signaling to ubiquitinmediated NF- $\kappa$ B activation," Molecular Cell, vol. 40, no. 1, pp. 63-74, 2010. 


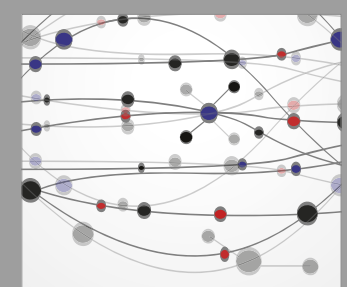

The Scientific World Journal
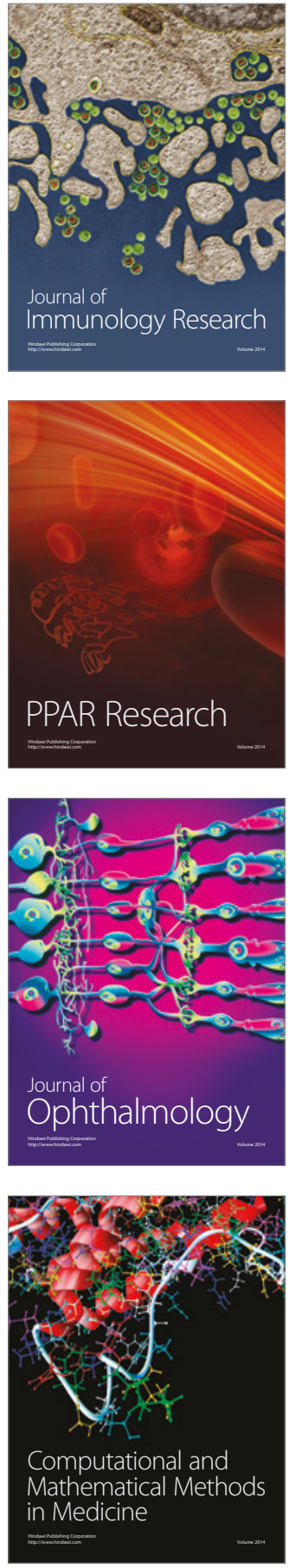

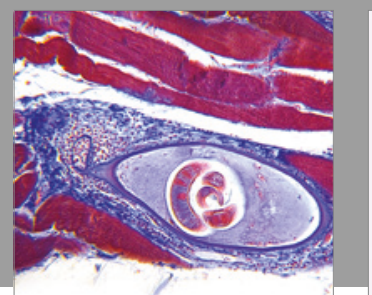

Gastroenterology Research and Practice
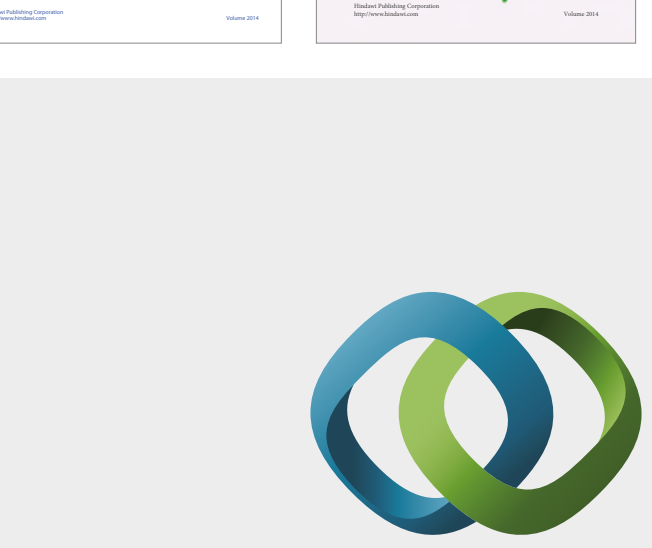

\section{Hindawi}

Submit your manuscripts at

https://www.hindawi.com
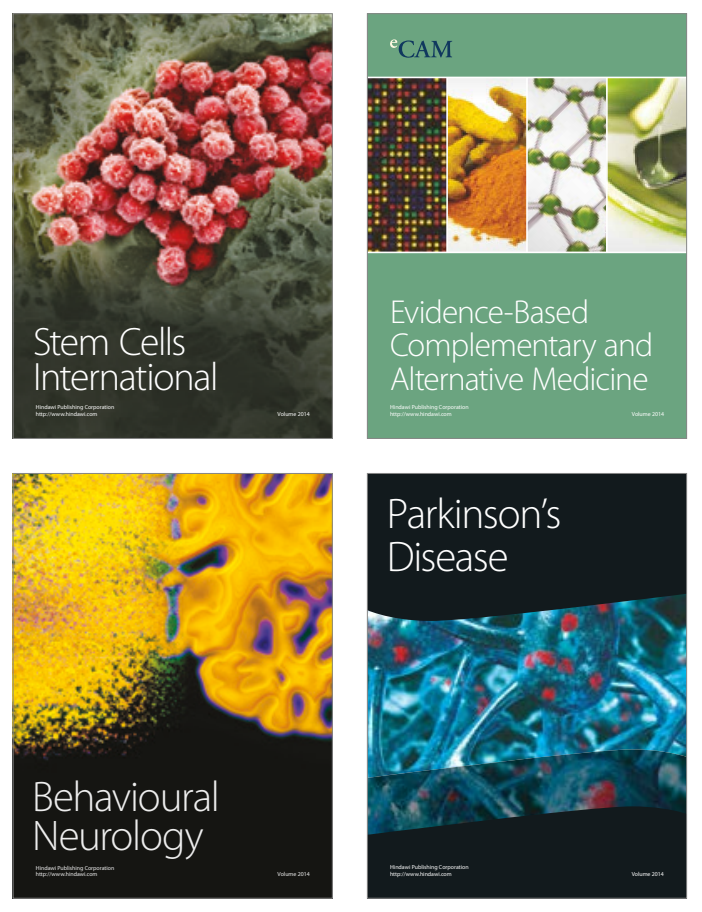
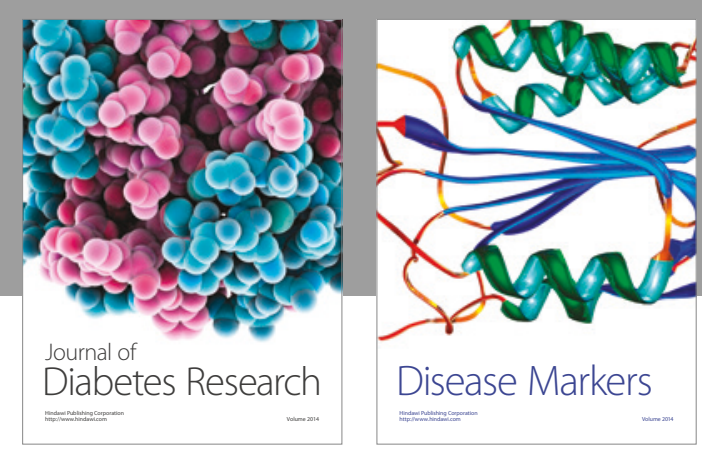

Disease Markers
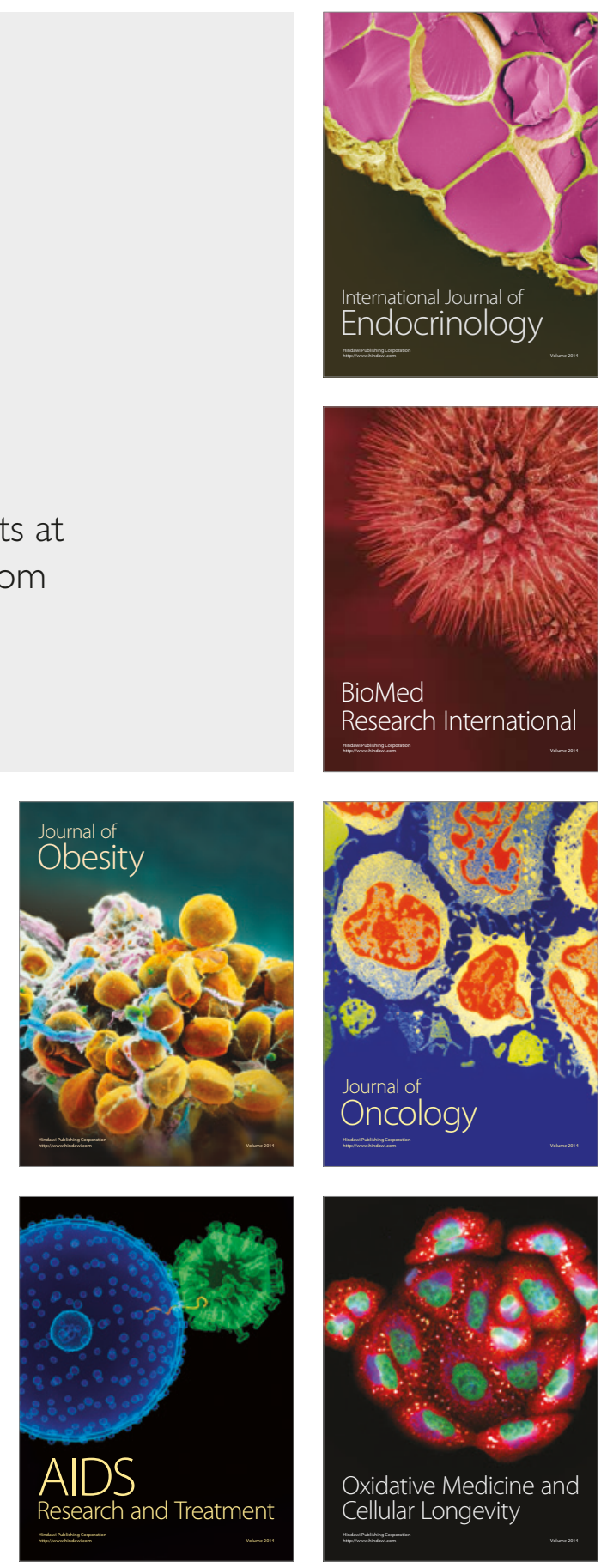